\title{
ORIENTATION EFFECTS AND SEYFERT CORES
}

\author{
R. P. NORRIS \\ Australia Telescope National Facility \\ PO Box 76, Epping, NSW2121, Australia
}

AND

A. L. ROY

Australia Telescope National Facility, University of Sydney, and NRAO

\section{Introduction}

Activity in galaxies takes on a bewildering array of guises. Not all of this diversity comes from fundamentally diverse physics, but if we can understand the relationships between different types of galaxy then we may be able to perceive the underlying physics. This taxonomic approach aims to determine which properties are common to several types of galaxy, and which properties differ. The danger in this process is that it is easy to invent spurious relationships between similar, but quite distinct, types of object.

We may compare the state of extragalactic astronomy to that of stellar astronomy around the turn of the century, when astronomers were trying to understand the relationships between the different types of star. When Hertzsprung \& Russell chose to plot colour against luminosity, then the main sequence, and other evolutionary groups, became clear, and subsequently enabled the physics of stars to be determined.

In extragalactic astronomy, we would like to know which axes to plot in order to reveal the underlying simplicity which will guide us to the physics. However, unlike the case of stars, the extragalactic H-R diagram will almost certainly not be two-dimensional - it is easy to list at least six axes which we know from our physical understanding to be independent.

However, we do know that at least one of these axes will represent orientation, since galaxies are not spherically symmetric. Orientation is particularly simple and has enormous predictive power, and so we need to understand the effect of orientation, so that we can disentangle it from 
other axes which may have deeper physical significance. The principal aim of this paper is to study a few of the relationships between different types of galaxy, and, in particular, the effect that orientation may have on Seyfert galaxies.

\section{Do Sy1 and Sy2 differ only in orientation?}

Seyfert galaxies can be grouped into two broad classes: Seyfert 1 (Sy1) and Seyfert 2 (Sy2). The observational differences between them are primarily that Sy1s have broader permitted lines and a stronger nuclear continuum than Sy2s. A naive interpretation might be that Sy1s are simply more energetic than Sy2s, but this is inconsistent with properties such as farinfrared (FIR) or radio luminosity, which are similar for both Sy1s and Sy2s.

An alternative explanation is that all Seyfert galaxies contain a nucleus, consisting of a compact continuum source and a broad line region (BLR), surrounded by a dusty torus. When viewed from above the plane of the torus, the broad permitted lines from the BLR and the optical continuum from the nucleus are both visible, and the galaxy is classified as a Sy1. When viewed from within the plane of the torus, the torus obscures our view of the nucleus at optical wavelengths, and the galaxy is classified as a Sy2. An extreme version of this model proposes that starburst galaxies are Seyfert galaxies, the narrow-line region (NLR) of which is also obscured, so that all we observe is the starburst emission in the outer part of the galaxy.

To test this hypothesis, Roy et al. (1994) examined the compact radio cores in carefully matched samples of $157 \mathrm{Sy} 1$ and Sy2 galaxies, using the Parkes-Tidbinbilla Interferometer (a $300-\mathrm{km}$ radio-linked interferometer). Since the dusty torus should be transparent to radio waves, the unified orientation model predicts that an equal number of cores should be seen in Sy1 and Sy2 galaxies. Alternatively, if Sy1s are more energetic versions of Sy2s, or if relativistic beaming were significant, then we should see a greater number of cores in Sy1s than in Sy2s.

The experiment gave a completely unexpected result: significantly more cores were seen in Sy2s (with a $48 \%$ detection rate) than in Sy1s (with a $26 \%$ detection rate). This firm observational result was at variance with any existing theory, and so demanded an explanation. Roy et al. proposed a variation of the unified orientation model. In this variation, the clouds in the narrow- line region may be optically thick at centimeter wavelengths, and so obscure our view of the radio emission from the compact core. An alternative model proposed by J. Miller (private communication) is that clouds in the broad-line region are optically thick, with the same effect. 


\section{The role of starburst activity in Seyferts}

Normal spiral and starburst galaxies show a tight correlation between their radio and FIR luminosity (e.g. Wunderlich et al., 1987). This correlation is remarkably tight and extends over five orders of magnitude in luminosity. While its cause is still not completely understood, it cannot be accounted for by selection effects or bias. It is almost certainly the result of the star formation process, and can therefore be used as an indicator of star formation activity.

Norris et al. (1988) showed that Seyfert galaxies also roughly obey this correlation, although they have a greater scatter about the correlation than do starbursts or normal galaxies. This suggests that the radio emission from Seyfert galaxies may be dominated by starburst activity. This view is supported by other evidence (e.g. Mouri \& Taniguchi 1992) that Seyfert activity is often accompanied by starburst activity.

Baum et al. (1993) have imaged a number of Seyfert galaxies at radio wavelengths. They found that, while the central jets appear to be oriented randomly with respect to the host galaxy, the broad outer radio lobes tend to be oriented perpendicularly to the host disc. This indicates that buoyancy may play a role in determining the direction of the lobes. In this respect, and in their overall morphology, these outer Seyfert lobes resemble the starburst-driven "superwinds" seen in some starburst galaxies (Heckman et al. 1987; Unger et al. 1989).

Baum et al. also showed that, for those Seyferts whose radio emission exceeded that predicted from the radio-FIR correlation, the excess radio emission was largely accounted for by the nuclear (sub-kpc) region. This implies that the outer lobes of these Seyfert galaxies follow the standard radio-FIR correlation. For the ratio of radio-to-FIR luminosity to be the same for these lobes as for starburst galaxies would be very surprising if they were driven by a different mechanism. Therefore, this offers some support for these outer lobes being driven by starburst rather than Seyfert activity. Roy et al. (1995), on the other hand, show that the inner jets do not follow the radio-FIR correlation.

Thus there is some support for the idea that while the inner jets of Seyfert galaxies are indeed tightly coupled to the Seyfert activity, the lobes in the outer parts may be driven by starburst rather than Seyfert activity. An alternative hypothesis is that there is some common underlying mechanism that makes two different energy sources follow the same relationship, and behave in similar ways. However, we should be cautious of naive attempts to unify Seyferts with radio galaxies and radio-loud quasars, as some of the properties of Seyferts may be dominated by starburst activity 


\section{The radio-loud/radio-quiet connection}

Although spiral galaxies and starburst galaxies lie on the well-known radioFIR correlation, many elliptical radio galaxies and radio-loud quasars have an excess of radio luminosity which places them well off this correlation. None of the unified schemes has yet successfully explained why galaxies fall into these two different groups - the radio-quiet galaxies, most of which are spirals, and the radio-loud galaxies, all of which are ellipticals. Particularly striking is the absence of a single known radio-loud spiral galaxy.

In an attempt to explore the transition from radio-quiet to radio-loud objects, we have cross-correlated the Parkes-MIT-NRAO 6-cm survey (Wright et al. 1995) with the IRAS point source catalogue (Roy \& Norris 1995), and have produced a pilot sample of 20 radio-loud, gas-rich objects. These include a BL Lac object, six Seyferts, and four spiral galaxies. Particularly striking is $00182-7112$, which is a Sy2 with a $0.1 \mathrm{Jy}$ VLBI core at $\mathrm{z}=0.3276$. It appears that this group includes radio-excess spirals, which may be transition objects between the radio-loud and radio-quiet classes of galaxy.

\section{Conclusion}

Our studies of the relationships between various types of galaxy show that:

- Sy1/Sy2 core detection rates are inconsistent with the standard orientation unification model (or any other model), but we can reconcile them by invoking a high NLR optical depth;

- Seyferts roughly follow the radio-FIR correlation, and in many cases the radio emission from Seyferts is dominated by starburst activity. Some Seyfert-like features may instead be due to starburst activity;

- there exists a class of intermediate objects between radio-loud and radio-quiet, including some "radio-loud Seyferts", which may represent a transition from radio-quiet to radio- loud objects.

\section{References}

Baum, S. A., O'Dea, C. P., Dallacassa, D., de Bruyn, A. G., \& Pedlar, A., 1993, ApJ, $418,553$.

Heckman, T. M., Armus, L., \& Miley, G. K., 1987, AJ, 93, 276.

Mouri, H., \& Taniguchi, Y., 1992, ApJ, 386, 68.

Norris, R. P., Allen, D. A., \& Roche, P. F., 1988, MNRAS, 234, 773.

Roy, A. L., Norris, R. P., Kesteven, M. J., Troup, E. R., \& Reynolds, J. E., 1994, ApJ, 432, 496.

Roy, A. L., \& Norris, R. P., 1995, MNRAS, submitted.

Roy, A. L., Norris, R. P., Kesteven, M. J., Troup, E. R., \& Reynolds, J. E., 1995, in preparation.

Unger, S. W., Pedlar, A., \& Hummel, E., 1989, $A \& A, 208,14$.

Wright, A. E., Griffith, M. R., Burke, B. F., \& Ekers, R. D., 1995, ApJS, 97, 347.

Wunderlich, E., Klein, U., \& Wielebinski, R., 1987, $A \& A S, 69,487$. 